and the previous contamination of the blood by bad air, bad water, bad food-this general "cholerine" may be owing to the diffusion of the epithelial cells in the air, and the mildness of the majority of cases to the smallness of the dose.

As Nature's operations are simple and uniform, we should expect her to act by the same neans in the same class of diseases; and that those contagious diseases in which the skin is affected, and those in which the mucous membranes of the lungs and of the intestines are the diseased tracts, would convey their contagion also by the epithelial cells of the skin or of the mucous membranes-that measles, small-pox, erysipelas-in all of which the vital properties of the inflamed skin are highly excited, and consequently the growth of the epithelial cells largely increased-would convey their contagion by the epithelial cells of the skin; whilst influenza, pertussis, catarrh, would be propagated by the epithelial cells of the respiratory track, and typhoid fever by those of the digestive track. The analogies between typhoid fever and cholera, the poison of both being conveyed by the secretions from the bowels, have been proved by Dr. W. Budd. Another striking analogy would be the conveyance of the poison in both cases by the epithelial cells of the intestines.*

Thus morbid epithelial cells of the skin would produce contagious skin-diseases; of the lungs, bronchial affections; of the intestines, diseases of the intestinal mucous membrane, like cholera and typhoid fever.

To sum up. It is admitted that the poison of scarlatina is conveyed by the epithelial cells of the skin, which desquamates freely, and that these dry cells retain their active power for some time, and when introduced into the body may propagate the same disease. In cholera, as in scarlatina, a similarly abundant desquamation of epithelial cells takes place, not from the skin, but from the intestines; and as it is by the excreta loaded with these cells from the intestines that the poison of cholera is chiefly conveyed, the inference is fair that, in cholera as in scarlatina, the poison is conveyed by epithelial cells.

I am not unaware of the deficiency of this view in proof sufficient to establish a fact relating to matter; and also of the difficulties in bringing such evidence in vital processes. It is an hypothesis explaining the several facts of contagion, and in accordance with our more advanced knowledge of cell pathology. But Lord Bacon said "Prudens quæstio dimidium scientiæ"; or, as Coleridge rendered it, "the forethoughtful quæry is the prior half of the knowledge

\section{PARALYTIC ECTROPIUM SUCCESSFULLY TREATED BY OPERATION.}

By Haynes Walton, Esq., Surgeon to the Central London Ophthalmic Hospital, and to St. Mary's Hospital.

Ectropium, or the turning out of an eyelid, is certainly one of the serious affections of the ocular appendages; but it is by no means so common as the opposite state, entropium, or the turning in of the lid. In aggravated cases, and especially when both eyelids are everted, the eyeball may suffer from exposure and want of necessary moisture. In the ordinary, or less severe states, and where only one

* In the loose secretions from the bowels of a patient in advanced typhoid fever, which I examined to-day, there were nucleated cell. in abundance, with columnar epithelium. For the sake of science, may I express the hope that Dr. I. Beale will extend his searching examinatious to the intestinal seoretions in typhoid fever. eyelid is everted, disfiguration and flowing of the lacrymal secretion over the cheek are the immediate evils. But in every degree there is a remote risk of injurious effects of inflammation of the eyeball, from inahility of the eyelids to wipe off or brush aside intruding particles. The exposed palpebral conjunctiva is always unnaturally and highly vascular, and so is frequently the ocular also.

The causes of ectropium may be referred to three classes. The first class includes abscesses about the orbit, usually at the circumference; burns, scalds, chemical injuries, ulcerations, either simple or specific, as from syphilis, lupus, sloughing after erysipelas, wounds, contusions, and surgical operations.

The second class includes eversion from disease, and thickening of the conjunctiva without tarsal disease.

The third class is ectropium from palsy of the portio dura-hemiplegia fascialis, by which the orbicularis palpebrarum muscle, among those that are palsied, no longer acts, and the power of closing the eye is lost; the upper eyelid cannot be depressed, while the lower falls down and turns outwards, becoming more everted in process of time. There are degrees of the paralysis here, just in fact as is witnessed in paralytic affections in other parts of the body.

The paralytic ectropium, the only one of which I shall treat, is the rarest of all. I am induced to make it the subject of a short communication, because I have lately treated a marked example most successfully by operative surgery; and I am not aware of any recorded instance of similar practice; nor do I know of any case having been so treated.

A gentleman, aged 24, was sent to me by Mr. R. Reid, in January of this year, on account of a distressing and increasing ectropium of the left lowerco eyelid from facial paralysis on that side, which oc-
curred in childhood. It is unnecessary to speak of the condition of the face. The ectropium produced much deformity, as the margin of the eyelid was very much depressed, and the conjunctiva was thickened and projecting, and very vascular. But a more annoying result was the constant flow of tears and mucous secretion over the cheek, roughness of the skin, and some excoriation.

After a short examination I was convinced that I could render essential benefit, and my patient readily assented to my proposal of treatment.

Chloroform having been given, I removed a strip of the diseased conjunctiva along the entire length of the eversion; and I may mention that I effected this by making two incisions with a scalpel, in the form of an ellipse, and dissecting away the isolated bit. It is by the contraction that ensues from this loss of substance that the eyelid is braced up, and in ordinary cases of ectropium I generally excise as much of the conjunctiva as is permanently exposed, and that effects the desired end. But here the lengthening of the tarsus, and the total loss of muscular support to it, required something more to be done; and also, the undue raising of the upper eyelid was another obstacle to success. To overcome these complications, I shortened both tarsi by removing a portion of each at the outer canthus, taking away conjunctiva as well, and brought the wound together by stitches.

It is not necessary to give a detailed account of the progress. It will answer every purpose merely to tell that the repair was rapid, and as effectual as it was possible. The eyelids are nicely bound up, and the stare arising from the prominence of the eyeball, and the exposure of it is almost overcome; so little indeed remains, as not to be noticed by a casual observer. The punctum lacrymale in each 
eyelid having been returned to its proper position, the tears are thoroughly conveyed away through the proper channels. Withal, there is no trace nor mark of the use of the scalpel.

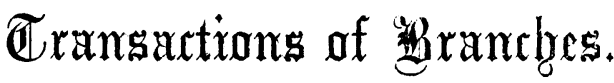

\section{SOU TH-W ESTER N B R A N C H.}

ON RECENT IMPROVEMENTS IN SURGERY.

By William Paú. Swain, Esq., Surgeon to the Royal Albert Hospital, Devonport.

[Read June 20th, 1866.]

IN the very short space of time which is allotted to me, it cannot, of course, be expected that the remarks I am about to address to you should be of a very elaborate nature, or that they should range over a large number of subjects. I shall endeavour to lay before you, as concisely as possible, some of the most recent improvements in surgical procedure, illustrating my remarks, as.far as I can, by the exhibition of the surgical instruments to which reference will be made.

Noticing first in order ophthalmic surgery, I would draw your attention to the suction-curette, introduced by Mr. Pridgin Teale, jun., of Leeds, by which soft cataracts, either congenital or traumatic, are removed with great facility. The pupil being dilated with atropine, the anterior capsule is lacerated with a needle, an opening is then made at the edge of the cornea safficiently large to admit the point of the curette, and the lens-matter sucked out. The results, after the use of this instrument, are most satisfactory.

The operation for extraction of the lens by flapoperation is less frequently performed than it was. The modification made by $\mathrm{Mr}$. Bowman and $\mathrm{Mr}$. Critchett in Schuft's spoon, enables the operator to remove even hard amber senile cataracts by traction. The old shaped spoon was of such dimension that it was difficult to insinuate it behind the lens, without rupturing the hyaloid membrane, and thus allowing vitreous humour to escape. The iris was also frequently brnised, and other damage inflicted on the tissues of the eye, which often resulted in the unfavourable termination of the case. The scoop now used is of much smaller dimensions, and avoids all the evils I have mentioned. The advantages this operation possesses over the old Hap-extraction, are, that chloroform may be given with safety, and that confinement to bed is seldom required after the second day, as the corneal wound heals very rapidly.

Passing on to the consideration of general surgery, I would call your attention to some cases of excision of the entire tongue, or a portion of it, which have been performed by Mr. Syme of Edinburgh and Dr. Buchanan of Glasgow. Some eighteen months ago, Mr. Syme removed the entire tongue from a patient affected with epithelial cancer of that organ. Twelve months after the performance of this operation, Mr. Syme reports, that the patient is in perfect health, can articulate well, sing without difficulty, and swallow finely divided food and fluids with great ease.

Dr. Buchanan, twelve months ago, excised one lateral half of the tongue with perfect success; and reports a case of excision of the entire organ, the patient sinking on the ninth day from pyæmia. Cancer of the tongue is one of the most fearful maladies with which the surgeon has to contend. I hardly know of any disease which brings such horrible concomitants in its train. Constitutional and topical remedies alike fail to arrest its progress. A lingering and a painful death is its sure result. To surgeons, then, and especially to those in charge of hospitals, it is a matter of congratulation, that the knife does sometimes effect a cure; and I trust that further experience of this operation will warrant its more frequent application.

Another form of disease, not so frequently met with, but most painful and distressing in its effects, is closure of the jaws by rigid cicatrices in and about the mouth, the result frequently of sloughing after fever. This condition has been remedied by $\mathbf{M r}$. Holt, of the Westminster Hospital, in one case, by simply separating the tissue of the cheek from the lower jaw, and sawing through a dense mass of new bone which had been thrown out between the alveoli of the apper and lower jaw; the adhesions being prevented from again taking place by the introduction of metal shields. In another case, Mr. Heath, of the same hospital, performed the operation advocated by Professor Esmarch of Kiel; viz., that of cutting out a wedge-shaped piece of bone and forming a false joint. I saw this case at Plymouth not long ago, and can testify to the satisfactory amount of movement the girl now possesses.

Laryngoscopy has now been sufficiently before the profession to require little remark from me. I introduced the subject in order that I may show you the instrument I am in the habit of using, which $I$ believe to be the simplest and best. Dr. G. Johnson has kindly lent me the small instrument he uses to remove pendulous tumours. The tumour is caught in a loop of fine wire, and by drawing back the trigger it is strangulated and torn off.

Before quitting the throat, I would just show you this tracheotomy-tube, which I have found very easy to introduce. I believe the most difficult part of the operation of tracheotomy is the introduction of the tube through the incision in the trachea. In this tube, when the inner one is removed, the outer one may be pinched up to a fine wedge-shaped point, which very much facilitates its introduction. You have doubtless seen reported in the Lancet of June 9th a curious crse of death from hømorrhage on the fifth day after tracheotomy; the hæmorrhage being produced by ulceration into the innominate artery. The accident was caused by the inner tube presenting a sharp point to the trachea, which caused the ulceration. In the tube I show you the edges are so turned that an accident of this kind could not happen.

The question of tracheotomy in croup is one which, I have no doubt you will agree with me, is very difficult to decide. I cannot, of course, now enter apon it fully ; but I would suggest that, in cases of rapid exudation, where air very imperfectly enters the lungs and symptoms are urgent, the insertion of a tube into the trachea frequently affords rapid relief and the only chance of salvation for the patient.

In amputations of the lower extremity, many improvements have taken place. The application of compression to the abdominal aorta, in amputation at the hip-joint, has deprived that formidable operation of its great danger; viz., hæmorrhage in the posterior flap from branches of the internal iliac. A clamp has been invented by Professor Lister of Glasgow, by which the circulation through the abdominal aorta may be completely controlled. In a case of my own, where I amputated at the hip-joint for encephaloid disease, the abdominal aorta was very effectually compressed by hand; but the use of this 